\section{An Unusual Major Fatty Acid Component in Human Depot Fat}

THE technique of gas chromatography has shown that naturally occurring fats and waxes contain saturated and unsaturated fatty acids with both even and odd numbers of carbon atoms. So far, however, the acids with odd numbers of carbon atoms have been reported to occur in only relatively small amounts in natural materials.

During analysis of human depot fat being carried out at this Institute, one specimen was shown to contain $8 \cdot 7$ per cent of a branched chain fatty acid containing thirteen carbon atoms. Other specimens of depot fat we have examined have always contained less than 0.1 per cent of this acid. The analysis showed no other abnormal features. The fat was obtained from the abdominal wall of a male African of the Sukuma tribe, whose approximate age was 60 years, admitted to hospital suffering from a strangulated hernia. The material was treated by the method described by Kingsbury et al. ${ }^{1}$ and $0.1 \mu \mathrm{l}$. of the resulting methyl esters was applied to a 4 -ft. column of 20 per cent 'Apiezon $L$ ' grease on 100-120 mesh 'Celite' held at a temperature of $186^{\circ} \mathrm{C}$. Argon was used as the carrier gas and the effluent gas was passed through a Pye ionization detector. The methyl ester of the component emerged from the column with a retention volume of 0.256 relative to methyl palmitate. With these conditions the relative retention volume of $n$-tridecanoic acid was $0 \cdot 307$. The amount of material available was too small to allow the nature of the acid to be determined accurately, but as the peak corresponding to the acid still appeared on the trace after bromination by the method of James and Martin ${ }^{2}$ it must be considered to be a saturated acid.

We are unable to explain the presence of the large amount of this acid in this specimen, but since it is most unlikely to occur to any extent in the diet, we suggest that its presence is due to an unusual method of fatty acid synthesis in the body.

D. S. MCLaren
W. W. C. Read

East African Institute for Medical Research, East Africa High Commission, P.O. Box 162

Mwanza, Tanganyika.

${ }^{2}$ Kingsbury, K. J., Paul, S., Crossley, A., and Morgan, D. M., Biochem. $\mathcal{J}_{., 78,}, 541$ (1961)

'James, A. T., and Martin, A. J. P., Biochem. J., 63, 144 (1956).

\section{Blood Glucose-6-Phosphate in Heterozygous Carriers and Patients with Liver Glycogen Disease (Von Gierke's Disease)}

Hsia and Kot ${ }^{1}$ have reported that children with liver glycogen disease (von Gierke's disease) and their parents, presumably heterozygous for the disease trait, both have high levels of g]ucose-6-phosphate and fructose-6-phosphate in red blood, cells. If correct, this observation would have very important practical as well as theoretical implications. It would greatly simplify the diagnosis and study of this disease and would open the possibility of its control through a eugenic approach. However, Hsia and Kot presented no evidence that their analytical methods wore appropriate or that the materials they measured were in fact glucose-6-phosphate and fructose-6-phosphate. Furthermore, the values these authors cited for their normal control population (glucose-6-phosphate,
50-370 mgm. per $100 \mathrm{gm}$. red blood cells; fructose-6phosphate, $120-570 \mathrm{mgm}$. per $100 \mathrm{gm}$. red blood cells) are much higher than those reported by other investigators ${ }^{2,3}$ or expected from theoretical considerations. Accordingly, their findings seemed questionable. We have re-examined this problem with respect to glucose-6-phosphate, using a specific enzymic analytical method. Our results are in sharp disagreement with those of Hsia and Kot.

Samples of whole blood or sedimented erythrocytes were deproteinized with equal volumes of 7 per cent perchloric acid. The filtrates were neutralized with potassium hydroxide and aliquots were incubated with glucose-6-phosphate dehydrogenase and triphosphopyridine nucleotide (TPN). The procedure of Aisenberg ${ }^{4}$ was followed, except that $300 \mu \mathrm{gm}$. of TPN was used and the total volume of the incubation mixture was reduced to $0.5 \mathrm{ml}$. The reaction was conducted in microcuvettes. The procedure was applied to standard solutions of glucose-6-phosphate; production of reduced TPN was within 6 per cent of the expected amount. The method was then tested by determining glucose-6-phosphate in whole blood to which different amounts of glucose-6-phosphate had been added. The results are shown in Table 1. There was some loss of added glucose-6-phosphate, presumably due to catabolism during the interval between addition of the material and inactivation of enzymes in the blood. However, recoveries were in a satisfactory range and reproducibility was good, down to concentrations of about $1 \mathrm{mgm} . / 100 \mathrm{ml}$. Below this level, the procedure proved inaccurate because the increases in optical density during incubation were very small.

Table 1. Recovery of GLucose-6-Phosphate ADDED to Whole BLOOD

Manipulations were carried out at room temperature; time interval before addition of perchloric acid was about 2 min. In each experiment the glucose-6-phosphate concentration was determined in an
aliquot of blood to which none had been added and the recovery results were appropriately corrected

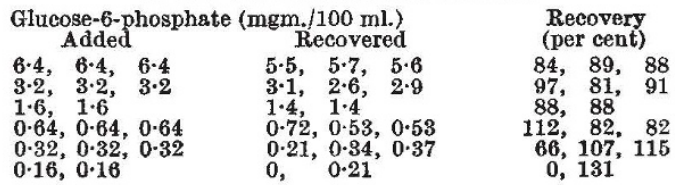

Table 2 presents the results obtained on whole blood and/or sedimented erythrocytes in 3 children with von Gierke's disease, 4 parents and 12 other blood relatives of such children, and 11 healthy adults without a family history of the disease. In all cases, the concentrations of glucose-6-phosphate were near the lower limit measurable by the method and much below those reported by Hsia and Kot. There were no major differences among affected children, their parents, other relatives, and normal controls. Because our analytical method was inaccurate at these low concentrations, however, it was not deemed worth

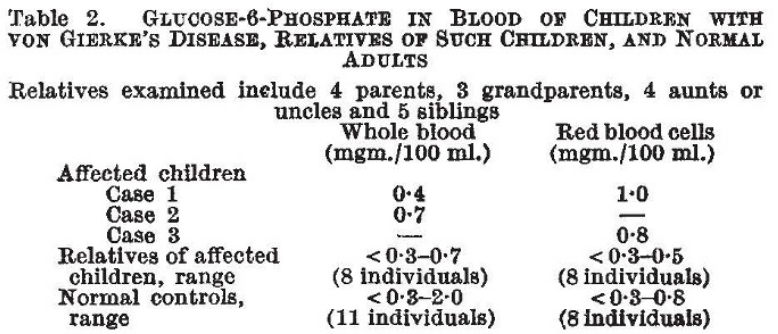

